\title{
Application of composite materials for the protection of sea shores and engineering structures against the impact of waves
}

\author{
E. S. Ashpiz ${ }^{1}$, A. O. Egorov $^{1}$ \& A. E. Ushakov ${ }^{2}$ \\ ${ }^{1}$ Moscow State University of Railway Engineering, Russia \\ ${ }^{2}$ ApATeCh - Company Applied Advanced Technologies, Russia
}

\begin{abstract}
The impact of sea waves creates considerable problems for the operation of the Tuapse-Adler railway which runs along the Russian Black Sea coast. The traditional means of protecting the railway against the impact of waves include pebble beaches, concrete seawalls, piers and shaped blocks. The disadvantages of today's concrete seawalls are high costs and long construction periods. To address these disadvantages a high-tech structure was developed made of composite materials which is lighter and more durable compared with traditional means.
\end{abstract}

Keywords: coastal erosion, calculation, railway embankment, seawalls, geogrid.

\section{Introduction}

Protecting sea shores against destruction by sea waves is still an issue of topical importance for many countries. In Russia, the most important area related to this issue is the Black Sea coast. The adverse effect of the sea creates problems for the Tuapse-Adler railway which stretches about 90 kilometers along the coast line [1], is subject to abrasion and has alongshore flows of pebble sediment [2]. In order to protect the railway, numerous engineering structures were erected, many of which have become defective over the years and need to be repaired. Moreover, at present a second rail track is under construction and its major part runs along the sea which creates the need to build new protective structures. It must be noted that the safety of railway traffic on this line strongly depends on 
natural factors, especially storms which in this region may reach a level of 8 points. Thus, reliable operation of coast protection structures will significantly reduce the risks that the city of Sochi, one of the country's major resorts and the capital of the 2014 Winter Olympic Games, will be cut off in terms of transportation.

\section{The existing system of coast protection structures}

The existing system of coast protection structures for this line includes pebble beaches, concrete seawalls, piers (made of stone or concrete blocks) as well as shaped blocks. An examination [3,4] of the condition of the bank protection constructions showed that many of the systems elements need to be overhauled and reinforced, fig 1.

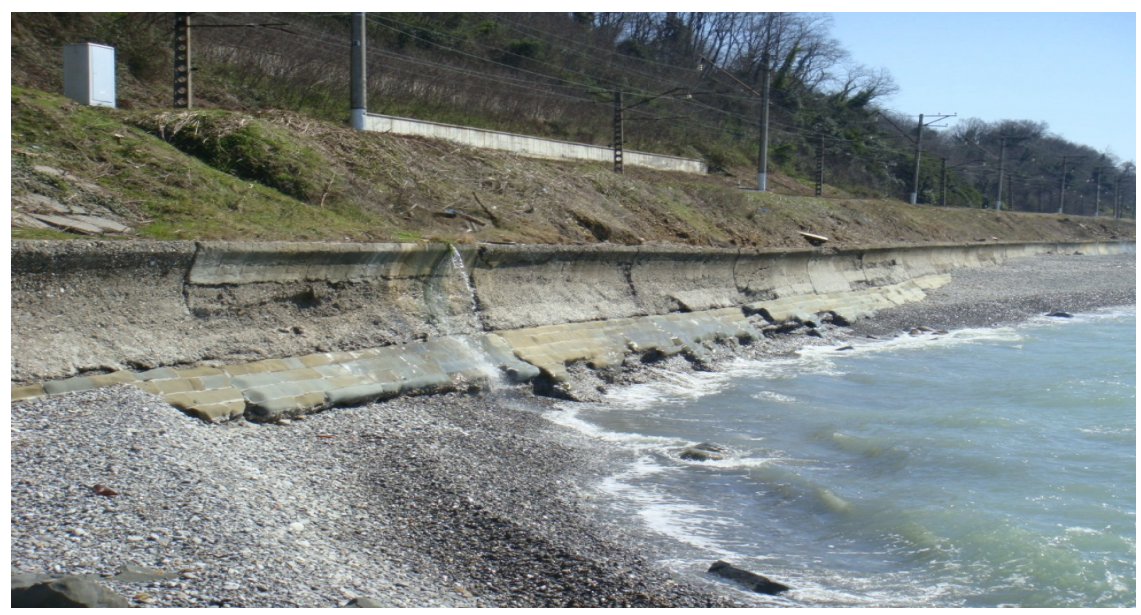

Figure 1: The condition of the coast protection structures at the TuapseAdler line. Dents in the bottom part of a seawall.

The existing concrete seawalls are one of the basic elements and they stretch along the railway line for about 70 kilometers. Up to $10 \%$ of these walls are in an unsatisfactory condition. At some points the bank protection constructions are completely destroyed, fig. 2 .

Investigation into the reasons for the collapse of the seawalls showed [5] that due to the underwashing of the concrete wall's foundation, individual concrete blocks shrank and some of them collapsed toward the sea. If there is sediment, which moves along the wall and no piers, the traditional concrete seawalls require additional protection with lining. Without lining, the sediment may cut a slot in the wall (fig. 3) throughout its width, normally $35-40 \mathrm{~cm}$ high and as a result the soil under the wall gets washed out and the wall topples toward the shore [6]. 


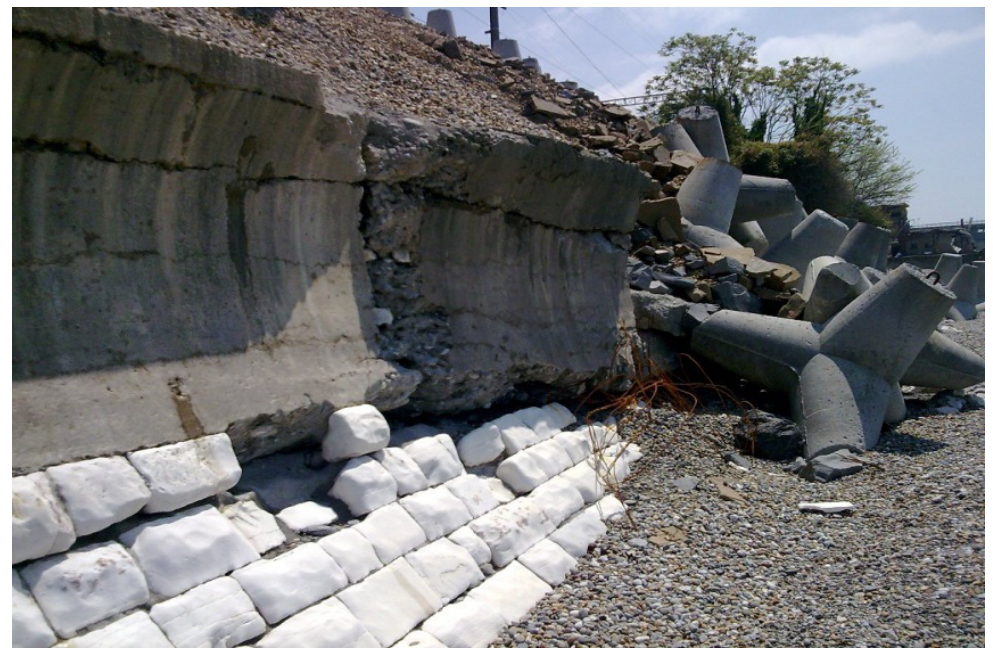

Figure 2: Seawall collapsed toward the sea. Yakornaya Schel Station.

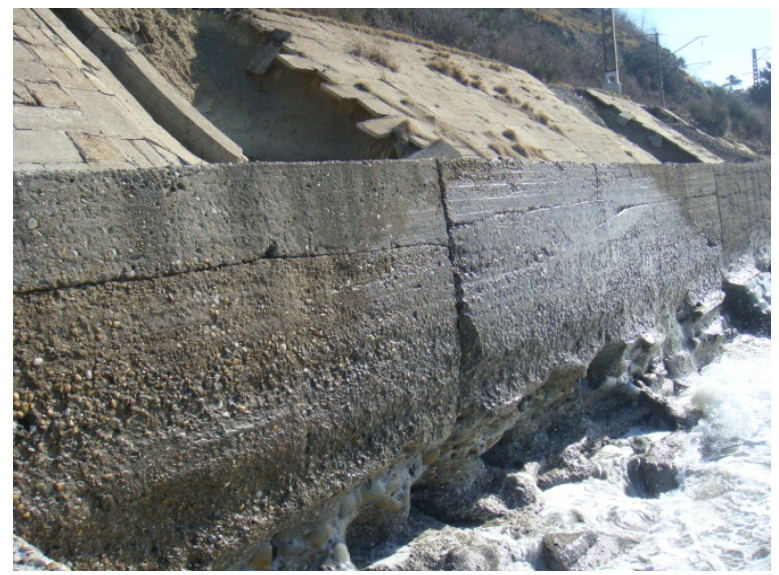

Figure 3: Reach-through slots in a concrete seawall.

Another disadvantage of replacing the existing concrete seawalls with similar structures is their high costs and long construction periods.

\section{Creating a new seawall structure}

In order to eliminate the drawbacks of the concrete seawalls, in 2008 Moscow State University of Railway Engineering and ApATeCh company developed a new design of a seawall made of composite materials which consists of 2 basic elements - a reinforced body of soil and fiberglass plastic panels joined together with the help of anchors. 


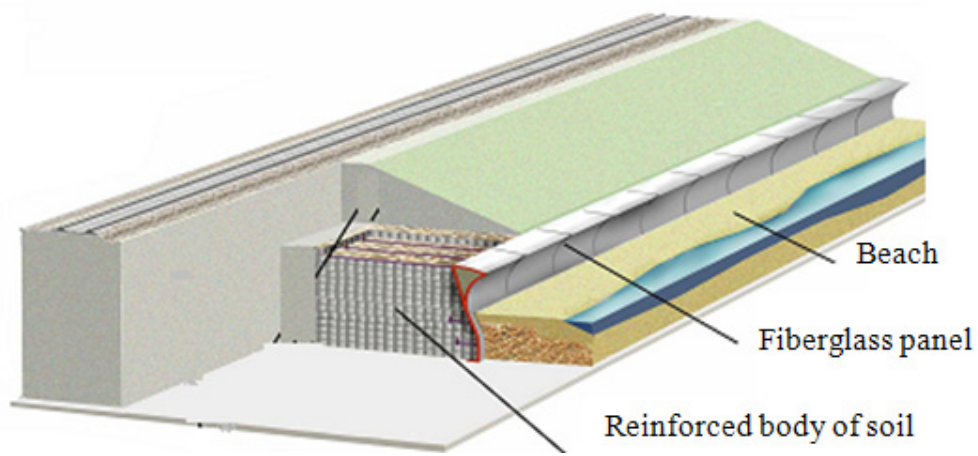

Figure 4: The structure of a seawall made of composite materials.

The structure was divided into two parts for accommodation of a combination of loads. The reinforced body of soil (on the side of the shore) accommodates the load from the railway vehicles and the weight of the soil and the fiberglass panel (on the side of the sea) accommodates the load from the sea waves and pebble suspension impact, protecting the reinforced body from being washed out.

\subsection{Fiberglass panels}

The fiberglass panels are installed on a beach with the embedding below sea level and have an inverted, wave resistant shape on the front side and flange joints on the back side, and the top parts of the panels bent toward the body of water are filled with porous material and encased in fiberglass covers fig. 5. Apart from that, in order to reduce sand and pebble erosion, the front surface of the seawall panels is covered with erosion-preventive coating. There are drain
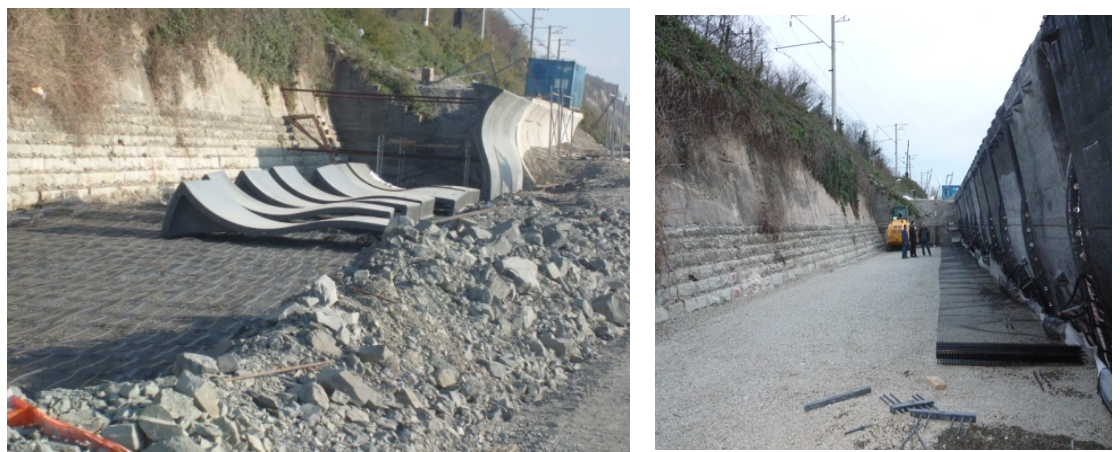

Figure 5: Fiberglass panels. The left-hand part shows installation of the fiberglass panels and the right-hand part shows the flange joints. 
holes in the bottom part of the fiberglass panels for derivation of ground water. To reduce the pressure of the body on the wall, the fiberglass covers are bent toward the shore at an angle, which is more than the fill soil internal friction angle.

Thanks to the physical properties of fiberglass panels their weight has been reduced and, consequently, the number of equipment for their delivery and installation has been decreased. For example, the weight of one running meter of a panel 4.0 meters high is $269 \mathrm{~kg}$, whereas the weight of a similar lightweight design concrete seawall is $\approx 8000 \mathrm{~kg}$.

\subsection{Reinforced body of soil}

In order to ensure secure attachment of the fiberglass panels to the reinforced body of soil some calculations were performed to test their strength against the dynamic load of waves' impact. As a result, the optimal interval at which the anchors connecting the wall with the soil at two levels $0.7 \mathrm{~m}$ and $1.6 \mathrm{~m}$ from the bottom of the wall was established. Reliability of the panels depends on the appropriate interaction of the panels with the reinforced body of soil which accommodates not only the load from the panels but also the loads transferred from railway vehicles and the embankment prepared for the second track

The reinforced body of soil is a backfilling reinforced by geogrids, fig.6. According to the technological requirement, the filling material for the body is pebble with a fraction of $20-40 \mathrm{~mm}$.
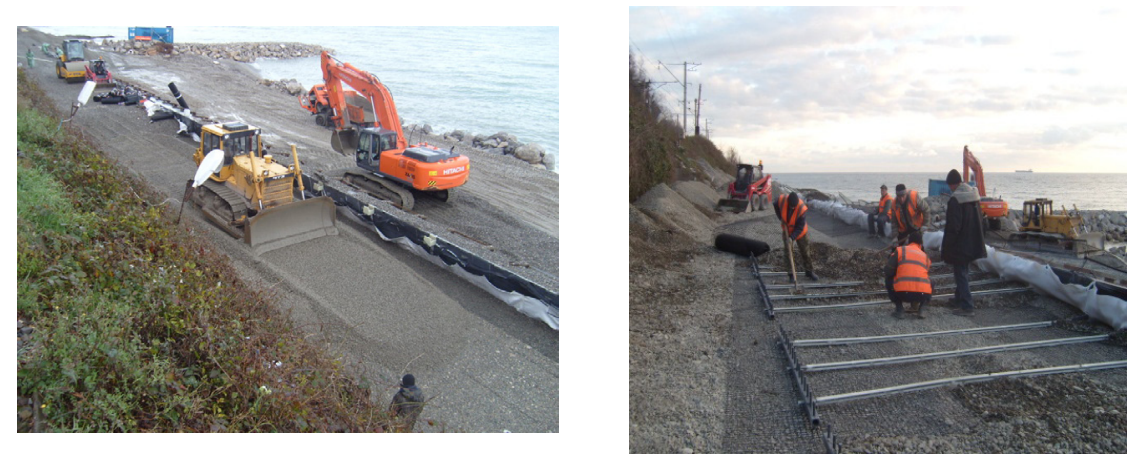

Figure 6: The layout of the Tensar SS-30 geogrid on the left, filling and grading of pebble with a fraction of $20-40 \mathrm{~mm}$ on the right.

The reinforced body of soil was designed with consideration of the results obtained by Tensar International Limited using Winwall 8.101 software. The calculation and design of the basic parameters of backfilling included the conditions of external and internal destruction of the body taking 8-point seismic activity into account according to the MSK-64 scale. Calculations were made for the following types of destruction: 
- external destruction:

- full collapse (circle slide of the structure and part of the escarpment with rotation);

- loss of foundation bearing resistance;

- shear of the construction relative to the soil (in-plane shear of the structure at the foundation);

- $\quad$ toppling of the structure;

- internal destruction:

- loss of reinforcement tensile strength;

- pulling reinforcement.

The following initial data was used for calculations:

- loading from a railway vehicle - $120 \mathrm{kPa}$ and from the weight of the upper construction $-17 \mathrm{kPa}$;

- operating temperature $10 \mathrm{oC}$;

- designed tensile strength of geogrids after 120 years of service life.

The following were approved for backfilling reinforcement on the basis of calculations:

- for the foundation below the composite panel - Tensar RE 80 uniaxial geogrid with an embedding length up to the existing wall and vertical interval of $0.5 \mathrm{~m}$;

- to ensure the local stability of pebble in the areas where the composite panel is attached to it as secondary reinforcement between the basic carcass use the Tensar SS 30 one-meter long biaxial geogrid. In the composite panel anchorage areas the length of the Tensar SS 30 geogrid shall be 2 meters.

The pebble backfilling shall be made layer by layer between the levels of the reinforcing geogrid. The primary filling layer between the Tensar 80RE geogrids shall be $50 \mathrm{~cm}$ thick. The technology of building seawalls made of composite materials allows construction to be carried out in cramped spaces and with very short deadlines, which is, without any doubt, an important advantage compared with the traditional structures.

\section{Monitoring the composite material seawalls}

In 2008, an experimental seawall was designed and built at one of the TuapseAdler second track construction sites, fig. 7. For the purposes of monitoring the condition of the composite material seawall, tensometric sensors were installed into the fiberglass panels, and ApATeCh employees read and analyzed their data a few times a month [7]. 

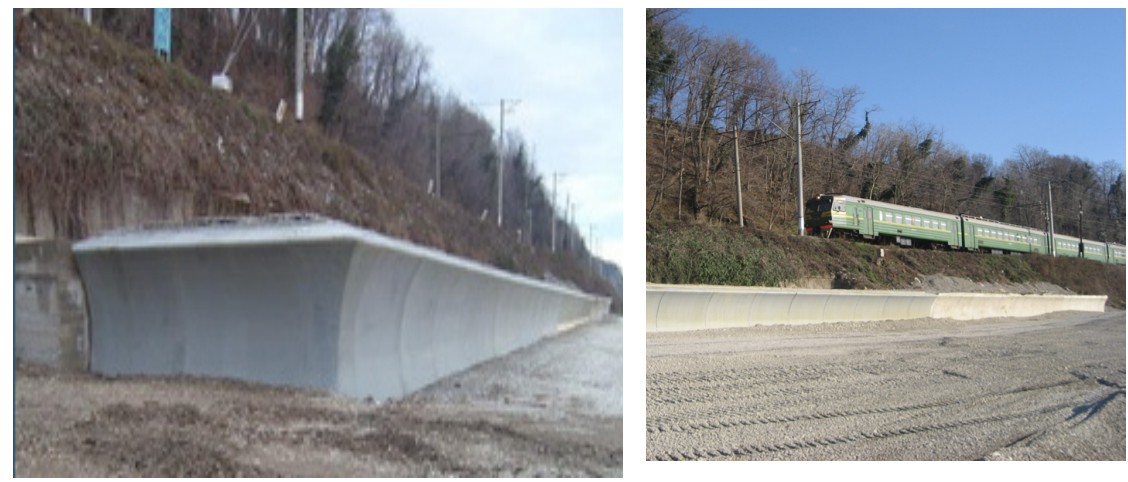

Figure 7: General overview of a composite material seawall.

\section{The main advantages of seawalls made of composite materials}

Thanks to modern technologies and materials, a high-tech seawall was created. Its basic elements are made of fiberglass plastic and polymers which are not affected by sea corrosion and decay.

\section{Conclusions}

1) A principally new design of sea wall made from a reinforced soil backing which absorbs stress from railway vehicles and the weight of the ground, and fibreglass panels which absorb stress from sea waves was developed.

2) The sea wall design is high-tech and can be constructed without the use of heavy construction equipment. This reduces the construction period by approximately 5 times.

3) The operational life of the sea wall is significantly increased thanks to the use of an erosion-preventative covering on the front part of the fibreglass panel.

4) In 2008, an experimental sea wall was designed and built at one of the construction sites for the second track of the Tuapse-Adler Line which is being constructed for the 2014 Winter Olympics in Sochi. It is currently being monitored.

\section{References}

[1] A.I. Panchenko, Shore-Protecting, Landslide-Protecting and RockfallProtecting Actions at the Site Tuapse-Adler of the North-Caucasus Railway. Proc. of the $2^{\text {nd }}$ Int. Conf. on Modern Issues of Design, Construction and Operation of Subgrade and Man-Made Structures. Elsevier: Moscow, pp.170-173, 2005 
[2] On-Site Research and Modeling of the Coastal Processes of the Black Sea Shore within the Krasnodar Territory of the Russian Soviet Federative Socialist Republic. Research Institute of Road Construction (ЦНИИС), Final Report GS-XI-85, book 2, pp. 12, 1985.

[3] V.V. Rudomsky. Personal communication, 25 February 2009, Head of railway department, Sochi, Russia.

[4] Examination of Defective Wave Resisting Structures at the site 1,913 km. PK1+00-1,951 km PK 8+00 of the Tuapse-Adler line of the North-Caucasus Railway. Moscow State University of Railway Engineering (MIIT), Report, book 2, pp. 6-30, 2009.

[5] Seawall, www.coastalwiki.org/coastalwiki/seawalls

[6] T.G. Smirnova, U.P. Pravdivets, G.N. Smirnov, Shore-Protecting Structures: Moscow, pp.132-133, 2002.

[7] ApATeCh, http://www.apatech.ru/seawall.html 